McLaughlin et al. (J. Biol. Chem., 248, $1466 ; 1973)$, and this is a case of particular interest, for it concerns the primitive eukaryote, yeast. The appearance of poly (A) in this cell suggests that it may be a common feature of all eukaryotes, and not only mammalian cells (though the absence of poly (A) from messenger specific for histones has been reported).

McLaughlin et al. followed the incorporation of label into the RNA, and have isolated the poly (A), after nuclease digestion of the rest of the chain, by adsorption on poly (T) coupled to cellulose. As a pretty demonstration that the poly (A) is a part of the messenger they have used a temperaturesensitive mutant, and shown that under nonpermissive conditions at $36^{\circ} \mathrm{C}$ the adenine label, previously incorporated at $23^{\circ} \mathrm{C}$, is displaced from the polysomes and sediments with free ribosomes. Moreover after dissociation of poly (A)-containing polysomes, all the poly (A) is found with the polydisperse messenger fraction. Another facet of this work is the application of a superior method of molecular weight estimation. Rather than merely use a sedimentation or electrophoresis procedure, with the hope that a calibration based on the usual RNAs will apply, they have performed end-group determinations. Chromatography of the product of alkaline hydrolysis, and comparison of the counts in adenylic acid and adenosine, showed in the first place that the poly (A) run was at the $3^{\prime}$ end of the RNA as in other eukaryotic messengers, and that the chain length was of the order of fifty nucleotides. An interesting additional observation is the failure of the entire messenger fraction to bind to the poly (T), and the absence of poly (A) from the part that does not bind. The integrity of the ribosomal RNA components indicates that this is not the result of nuclease activity, and it may present a parallel to the case of histone messenger. The variety of possible roles for the poly (A) tract of the messenger is now a subject of speculation, and no doubt new experimentation.

A variant of the end-group method has been used to identify poly (A) at the 3 ' end of the HeLa cell messenger and of heterogeneous nuclear RNA, by Nakazato, Kopp and Edmonds (ibid., 1472). Here the terminal sugar is oxidized with periodate, and the resulting glycol reduced with tritiated borohydride, so as to introduce an endgroup label. The bulk of this label is recovered in the nuclease-resistant poly (A) fraction. In addition, however, the heterogeneous nuclear RNA appears to contain a short run of poly (A), some twenty residues long, which is not by the same criteria at the $3^{\prime}$ end. The presence of the bulk of the poly (A) at this end of the nuclear RNA supports the view that the messenger comprises the $3^{\prime}$ end of the precursor.

\section{CONSERVATION \\ Sheep or Grouse?}

from our Plant Ecology Correspondent Conservation involves the practical management or manipulation of ecosystems in such a way that a predetermined ecological goal can be achieved. The goal itself is often selected on aesthetic, emotive, or economic grounds rather than scientific ones. A good example of this principle at work is found in the current issue of Biological Conservation $(5,41 ; 1973)$, in which Watson and O'Hare describe experiments designed to "improve" the Irish blanket bogs.

The experiments which they describe are aimed at increasing the red grouse population of the Donegal and Mayo bogs with a view to improving the shooting potential of the area and hence enhancing tourism. The experiments consist of fencing plots to exclude grazing animals and fertilizing them with superphosphates $\left(502 \mathrm{~kg} \mathrm{ha}^{-1}\right)$, potassium sulphate $\left(125 \mathrm{~kg} \mathrm{ha}^{-1}\right)$ and smaller quantities of other nutrients. Some plots were also drained. Two years after draining and fertilizing, heather (the staple food of the red grouse) had a productivity four times that in control plots and the phosphorus content of its tissue was twice that of the controls. The density of grouse in the treated areas had also increased by a factor of two and their breeding success was three times that of birds in control areas.
As a management system for increasing the density of grouse in the short term, drainage and fertilization must be considered a most effective treatment. The authors do not, however, stress the fact that these results were obtained in the absence of stock grazing. This is unfortunate, for the work of Watson in Scotland (Nature Conservancy Research in Scotland, Report for 1968-70, NERC) demonstrates that without the exclusion of grazing animals, fertilization produces no change in the population density of grouse. The choice, therefore, seems to be sheep or grouse.

There are other ecological questions which should be raised with respect to this work. Watson and O'Hare have found that heather under low phosphate conditions responds well to added phosphate. In natural ecosystems, however, a species which can survive, even at suboptimal growth, in a poor environment may be at a considerable advantage in avoiding competition from more aggressive species. Raising the nutrient status of a bog ecosystem could destroy this balance and lead to the displacement of heather by more nutrient-demanding, vigorous plants. The long-term effects of drainage and fertilization could be harmful, even to grouse. Eutrophication of many systems has a short-term bene ficial influence on productivity which is not maintained.

In western Ireland the economic consideration must be paramount in selecting an appropriate management system. The authors are unwise, however, in suggesting that such a system would also result in improved conditions for wildlife in general. This type of management is likely to benefit only the grouse and the shooting fraternity.

\title{
New Case of Disulphide Degeneracy
}

THE proteolytic enzyme, papain, as conventionally prepared, contains the active enzyme, with a free thiol group at position 25 in the chain, an inactive component, which can be activated by exposure to reducing agents, and a dead fraction in which the vital thiol is thought to be oxidized. By eliminating protective thiols from the preparation, Brocklehurst and Kierstan have isolated a fraction which contains some thiol groups, but is nevertheless inactive: they report their findings in next Wednesday's Nature New Biology (April 11).

Whereas the thiol group in active papain is highly reactive towards a disulphide reagent, dipyridyldisulphide, even at acid $p \mathrm{H}$, that of the inactive fraction behaves differently, the $p \mathrm{H}$ reaction rate profile corresponding to $p \mathrm{~K}$ of 7.6. When reduced this material is converted to normal active papain.
Reaction of the thiol groups with radioactive isodoacetamide, followed by reduction, showed that two different types of thiol groups, in respect of $p \mathrm{H}$ reactivity dependence, are present in the activated species. This can be interpreted to indicate that in the thiolcontaining inactive species, cys- 25 is involved in a disulphide bond with one of the other six half-cystines in the molecule, and that conversion to the active enzyme ensues by thiol-disulphide exchange.

A detailed activation scheme involving thiol-disulphide exchange between the active site cys- 25 and residues at positions 22 and 63 is suggested. Two other proteins with the rare property of containing both cystine and cystine residues, have previously been shown to exist in structurally degenerate states, in which alternative disulphide pairings occur. 